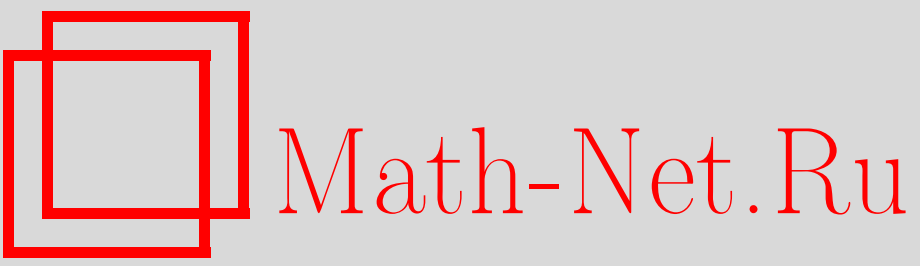

Ю. П. Чубурин, О попадании собственного значения (резонанса) оператора Шредингера на границу зоны, ТМФ, 2001, том 126, номер 2, 196-205

DOI: https://doi.org/10.4213/tmf424

Использование Общероссийского математического портала Math-Net.Ru подразумевает, что вы прочитали и согласны с пользовательским соглашением

http://www.mathnet.ru/rus/agreement

Параметры загрузки:

IP : 18.234 .197 .8

26 апреля 2023 г., 07:10:55 


\section{О ПОПАДАНИИ СОБСТВЕННОГО ЗНАЧЕНИЯ (РЕЗОНАНСА) ОПЕРАТОРА ШРЕДИНГЕРА НА ГРАНИЦУ ЗОНЫ}

Для оператора Шредингера с периодическим потенциалом, возмущенным функцией, периодической по двум переменным и стремящейся к нулю по третьей, получены условия попадания уровня (собственного значения или резонанса) на границу зоны. Обсуждается переход уровня через эту границу при изменении величины возмущения.

\section{1. ВВЕДЕНИЕ}

Пусть $V(x)$, где $x=\left(x_{1}, x_{2}, x_{3}\right) \in \mathbb{R}^{3}$, - вещественная функция из $L^{\infty}\left(\mathbb{R}^{3}\right)$, периодическая по всем переменным с периодом единица; $W(x) \in L^{\infty}\left(\mathbb{R}^{3}\right)$ - вещественная функция, периодическая по переменным $x_{1}, x_{2}$ с периодом единица и удовлетворяюшая неравенству $|W(x)| \leqslant C \exp \left(-a\left|x_{3}\right|\right)$, где $a>0, x \in \mathbb{R}^{3}$. Самосопряженные операторы Шредингера $H_{0}=-\Delta+V(x)$ и $H=H_{0}+W(x)$, действуюшие в $L^{2}\left(\mathbb{R}^{3}\right)$, будучи разложены в прямом интеграле пространств $L^{2}(\Omega)$, где $\Omega=[0,1]^{2} \times \mathbb{R}$ (см. [1], [2]), порождают семейства операторов $H_{0}\left(k_{\|}\right)=-\Delta+V(x)$ и $H\left(k_{\|}\right)=H_{0}\left(k_{\|}\right)+W(x)$, действуюших в $L^{2}(\Omega)$ и определенных на (достаточно гладких) блоховских по переменным $x_{1}, x_{2}$ функциях. Вектор $k_{\|}=\left(k_{1}, k_{2}\right) \in \Omega^{*}=(-\pi, \pi]^{2}$ называется квазиимпульсом и определяется условием блоховости. Под блоховскими по переменным $x_{1}, x_{2}$ функциями $\phi(x), x \in \mathbb{R}^{3}$, понимаются функции, удовлетворяюшие условию

$$
\phi\left(x+\left(n_{\|}, 0\right)\right)=e^{i\left(k_{\|}, n_{\|}\right)} \phi(x), \quad n_{\|}=\left(n_{1}, n_{2}\right) \in \mathbb{Z}^{2},
$$

а также сужения таких функций на $\Omega$. Заметим, что оператор $H\left(k_{\|}\right)$является оператором энергии электрона в ситуациях: 1) плоской кристаллической пленки (в случае, если $V=0$ ); 2) слоистой структуры; 3 ) поверхности кристалла (в случае, когда функция $V(x)+W(x)$ близка к нулю для $\left|x_{3}\right| \leqslant d$, где $d$ достаточно велико; поверхность при этом "удвоенная").

\footnotetext{
* Физико-технический институт УрО РАН, Ижевск, Россия
} 
Обозначим через $H_{0}(k)=-\Delta+V(x)$ оператор, определенный на блоховских по всем переменным функциях из $L^{2}\left(\Omega_{0}\right)$, где $\Omega_{0}=[0,1]^{3}$; здесь квазиимпульс трехмерен: $k=\left(k_{\|}, k_{3}\right) \in \Omega_{0}^{*}=(-\pi, \pi]^{3}$. Спектр оператора $H_{0}(k)$ дискретен (см. [1], разд. ХIII.16). Обозначим через $E_{n}(k)$ его собственные значения, занумерованные в порядке неубывания с учетом кратности, а через $\psi_{n}(x, k)$ - соответствуюшие собственные функции $(n=1,2, \ldots)$. Существенный спектр оператора $H\left(k_{\|}\right)$равен (см. [3], предложение 1$)$

$$
\sigma_{\mathrm{ess}}\left(H\left(k_{\|}\right)\right)=\sigma\left(H_{0}\left(k_{\|}\right)\right)=\bigcup_{k_{3} \in(-\pi, \pi], n=1,2, \ldots}\left\{E_{n}(k)\right\},
$$

где $\sigma\left(H_{0}\left(k_{\|}\right)\right)$- спектр оператора $H_{0}\left(k_{\|}\right)$, и имеет зонную структуру (состоит из промежутков).

Пусть собственное значение $E_{n_{0}}\left(k_{0}\right)=E_{0}$, где $k_{0}=\left(k_{\|}, k_{3}^{(0)}\right) \in \Omega_{0}^{*}$, невырожденное и находится на границе множества $\sigma\left(H_{0}\left(k_{\|}\right)\right.$) (на границе зоны). Согласно теории возмущений (см. [4], добавление 1$) E_{n_{0}}(k)$ аналитически зависит от $k_{3}$ в окрестности $k_{3}^{(0)}$; очевидно, что

$$
\frac{\partial E_{n_{0}}\left(k_{0}\right)}{\partial k_{3}}=0
$$

Здесь и далее $\partial^{m} E_{n_{0}}\left(k_{0}\right) / \partial k^{m}$ означает $\partial^{m} E_{n_{0}} / \partial k^{m}$ в точке $k_{0}$. Будем предполагать выполнение условия

$$
\frac{\partial^{2} E_{n_{0}}\left(k_{0}\right)}{\partial k_{3}^{2}} \neq 0
$$

кроме того, предполагаем, что $E_{n}\left(k_{0}\right) \neq E_{0}$ для $n \neq n_{0}$, т.е. граниша зоны описывается только функцией $E_{n_{0}}(k)$. Равенство $(1)$ определяет в силу $(2)$ в (комплексной) окрестности точки $k_{0}$ комплексно-аналитическоемногообразие $\mathcal{V}_{0}$, описываемое некоторой аналитической функцией $k_{3}=k_{3}^{(0)}\left(\tilde{k}_{\|}\right)$, заданной в (комплексной) окрестности точки $k_{\|}$.

Уравнение $E_{n_{0}}(k)=E$ имеет относительно $k_{3}$ для $E$, близких к $E_{0}$, ровно два корня (сливаюшихся при $E=E_{0}$ ), через $k_{3}^{(1)}$ обозначим тот из них, для которого (при вешественных значениях $\left.k_{3}^{(1)}, E_{n_{0}}\left(k_{\|}, k_{3}^{(1)}\right)\right)$

$$
\frac{\partial E_{n_{0}}\left(k_{\|}, k_{3}^{(1)}\right)}{\partial k_{3}}>0, \quad k_{3}^{(1)} \neq k_{3}^{(0)}
$$

В дальнейшем вместо величины $E$ будем часто использовать $k_{3}^{(1)}=k_{3}$.

Положим $R_{0}\left(k_{\|}, E\right)=\left(H_{0}\left(k_{\|}\right)-E\right)^{-1}, R\left(k_{\|}, E\right)=\left(H\left(k_{\|}\right)-E\right)^{-1}$. Согласно [3] функция Грина оператора $H_{0}(k)$ (т.е. ядро резольвенты оператора, возможно, продолженное аналитически по параметру $k_{3}$ (или по $E$ на второй лист)) имеет вид

$$
\Gamma(x, y, k)=-\frac{\psi_{n_{0}}\left(x, k_{0}\right) \overline{\psi_{n_{0}}\left(y, k_{0}\right)}}{i\left(k_{3}-k_{3}^{(0)}\right) \partial^{2} E_{n_{0}}\left(k_{0}\right) / \partial k_{3}^{2}}+\Gamma^{\prime}(x, y, k),
$$

где функция $\Gamma^{\prime}$ такова, что выражение

$$
\sqrt{W(x)} \Gamma^{\prime}(x, y, k) \sqrt{W(y)}
$$


является аналитической $L^{2}(\Omega \times \Omega)$-значной функцией аргумента $k_{3}=k_{3}^{(1)}$ (здесь и далее $\left.\sqrt{z}=\sqrt{\rho e^{i \phi}}=\sqrt{\rho} e^{i \phi / 2}, \phi \in(-\pi, \pi]\right)$. По переменной $E$ (вместо $k_{3}$ ) функция $\Gamma$ имеет в точке $E_{0}$ ветвление порядка 2 , что обеспечивает возможность появления вблизи $E_{0}$ резонансов. Уравнение, описьвающее как собственные значения (для $E \in \mathbb{R}$ ), так и резонансные уровни (или резонансы, см. ниже), имеет вид [3]

$$
\psi(x)=-\int_{\Omega} \Gamma\left(x, y, k_{\|}, E\right) W(y) \psi(y) d y .
$$

С помощью замены $\phi=\sqrt{W} \psi$ перейдем к эквивалентному (предполагаем, что $W \not \equiv 0$ ) “модифицированному” [1] уравнению, заменяя также $E$ на $k_{3}$ :

$$
\phi(x)=-\int_{\Omega} \sqrt{W(x)} \Gamma(x, y, k) \sqrt{W(y)} \phi(y) d y,
$$

которое будет рассматриваться в классе $L^{2}(\Omega)$. Под резонансами будем понимать значения $E$, отвечаюшие величинам $k_{3} \mathrm{c} \operatorname{Im} k_{3}<0$, для которых сушествует ненулевое решение уравнения (6) (ср. [3], [5]; заметим, что употребление здесь термина "резонанс" несколько условно, см., например, [6]). Условие на мнимую часть $k_{3}$ означает экспоненциальное возрастание функции Г и, следовательно, функции $\psi$ из уравнения (5); при этом $E$ находится на втором листе (см. [3]). Наоборот, неравенство $\operatorname{Im} k_{3}>0$ означает, что $\psi$ экспоненциально убывает и, следовательно, соответствующее $E$ является собственным значением.

Множество точек $\tilde{k}=\left(\tilde{k}_{\|}, k_{3}\right) \in \mathbb{C}^{3}$ из окрестности $k_{0}$, а также отвечающее им множество точек $\left(\tilde{k}_{\|}, E\right)$, для которых сушествует ненулевое решение уравнения (6), будем называть поверхностью уровней и обозначать через $\mathcal{V}$; при этом $E$ (уровни) могут быть как собственными значениями, так и резонансами. Множество $\mathcal{V}$ является аналитическим множеством в $U \backslash \mathcal{V}_{0}$, где $U$ - некоторая комплексная окрестность точки $k_{0}$, описываемым уравнением вида $\Delta(k)=0$, где $\Delta(k)$ - аналитическая функция в $U \backslash \mathcal{V}_{0}$ (и мероморфиная в $U$ ), не равная нулю тождественно (последнее справедливо, так как в противном случае $E_{0}$ не было бы границей зоны). Это следует из доказательства мероморфной теоремы Фредгольма [1] (интегральный оператор в правой части (6) в силу (3) и сказанного выше удовлетворяет условиям этой теоремы); см. также ниже доказательство теоремы 1 . Будем говорить, что $\mathcal{V}$ проходит через точку $k_{0}$ (границу зоны), если $k_{0}-$ предельная точка множества $\mathcal{V}$

В настоящей работе определено условие прохождения поверхности уровней $\mathcal{V}$ через $k_{0}$. Это представляется важным по той причине, что наличие уровня вблизи нулевой энергии вызывает, вообше говоря, особенности в рассеянии, причем здесь сушественную роль играет также характер зависимости уровня от квазиимпульса $k_{\|}$(см. [7]). Поведение же уровня в случае, если $\mathcal{V}$ проходит через точку $k_{0}$, весьма специфично (см. ниже теорему 3 и замечание 1 к ней). При введении "параметра связи" $\mu$ в потенциал заменой $W$ на $\mu W$ оказывается, что попадание уровня на границу зоны происходит “достаточно часто". Заметим, что это подтверждается также вычислениями, проведенными в работе [7]. Наконец, показывается, что в случае аналитической зависимости уровня от 
$\mu$ собственное значение при касании гранищы зоны отражается от нее и превращается в резонанс. Заметим, что в рассматриваемом случае функция Грина оператора $H_{0}\left(k_{\|}\right)$ имеет полюс на границе зоны, чего нет в случае возмущающего потенциала, убывающего по всем переменным [8], и потому в полученных результатах вместо не сушествуюшей в точке $k_{0}$ функции Грина фигурируют ее регулярная часть и вычет.

\section{2. ПРОХОЖДЕНИЕ ПОВЕРХНОСТИ УРОВНЕЙ ЧЕРЕЗ ГРАНИЦУ ЗОНЫ}

Лемма 1. Пусть резольвента $R_{0}(k)=R_{0}\left(k_{\|}, E\right)$ существует. Тогда существование оператора $\sqrt{W} R(k) \sqrt{W}$ әквивалентно существованию оператора $(1+$ $\left.\sqrt{W} R_{0}(k) \sqrt{W}\right)^{-1}$, причем имеет место равенство

$$
\left(1+\sqrt{W} R_{0}(k) \sqrt{W}\right)^{-1}=1-\sqrt{W} R(k) \sqrt{W} .
$$

Доказательство леммы стандартно.

Вследствие (3) и мероморфной теоремы Фредгольма [1] $\left(1+\sqrt{W} R_{0}(k) \sqrt{W}\right)^{-1}$ представляет собой мероморфную операторнозначную функцию переменной $k_{3}$ с полюсами в резонансах. Лемма 1 позволяет определить резонансы как полюсы мероморфной функции $R(k)$, продолженной равенством (7) по переменной $k_{3}$.

Обозначим через $A(k)$ оператор с ядром (4). Всюду в дальнейшем предполагаем, что либо $W(x) \geqslant 0$, либо $W(x) \leqslant 0$ для всех $x$. Далее, будем предполагать, что вблизи точки $k_{3}^{(0)}$ функция $E_{n_{0}}=E_{n_{0}}\left(k_{0}+\left(0,0, k_{3}-k_{3}^{(0)}\right)\right)$ является четной относительно переменной $k_{3}-k_{3}^{(0)}$. Так будет, например, в случае, если $k_{3}^{(0)}=0$, а $V(x)$ - четная по $x_{3}$ функция.

Под лакуной, как обычно, понимаем открытый промежуток между зонами (для крайней зоны - полубесконечный интервал).

ЛЕмма 2. Число $E=E_{n_{0}}(k)$ из малой окрестности точки $E_{0}$ принадлежстт лакуне, примыкающей $\kappa$ точке $E_{0}$, в том и только в том случае, если $k_{3}-k_{3}^{(0)} \in i \mathbb{R}$.

ДокАЗАТЕльство. Пусть для определенности $E_{0}$ - точка минимума, $k_{3}=k_{3}^{(0)}+i \epsilon$, где $\epsilon \in \mathbb{R}$ достаточно мало. Функция $E_{n_{0}}(k)$ разлагается в ряд Тейлора по четным степеням $k_{3}-k_{3}^{(0)}$, следовательно,

$$
E=E_{n_{0}}(k)=E_{0}+\frac{1}{2} \frac{\partial^{2} E_{n_{0}}\left(k_{0}\right)}{\partial k_{3}^{2}}(i \epsilon)^{2}+\cdots<E_{0},
$$

если $\epsilon \neq 0$. Наоборот, пусть $E_{n_{0}}(k)<E_{0}$ для малых $k_{3}-k_{3}^{(0)}$. Уравнение $E_{n_{0}}(k)=$ $E<E_{0}$, где $E$ близко к $E_{0}$, в силу подготовительной теоремы Вейерштрасса [9] имеет ровно два корня $k_{3}^{(1)}, k_{3}^{(2)}$. С другой стороны, функция $E_{n_{0}}=E_{n_{0}}\left(k_{0}+(0,0, i \epsilon)\right)$ имеет по $\epsilon$ максимум в точке $\epsilon=0$, и, таким образом, уравнение $E_{n_{0}}\left(k_{0}+(0,0, i \epsilon)\right)=E<E_{0}$, $\epsilon \neq 0$, имеет два вешественных корня $\epsilon_{1} \neq \epsilon_{2}$. Поэтому $k_{3}^{(1,2)}=k_{3}^{(0)}+i \epsilon_{1,2}$. Лемма доказана. 
Лемма 3. Если значение $E$, близкое $\kappa E_{0}$, принадлежст лакуне, то оператор $A(k)=A\left(k_{\|}, E\right)$ самосопряжен.

ДокАЗАТЕЛЬСтво. Оператор $R_{0}(k)$ самосопряжен в лакуне, поэтому $\overline{\Gamma(x, y, k)}=$ $\Gamma(y, x, k)$. Вследствие $(3)$ и вешественности $i\left(k_{3}-k_{3}^{(0)}\right)$ (см. лемму 2$)$ это же верно и для $\Gamma^{\prime}$, а в силу знакопостоянства $W$ - и для ядра оператора $A(k)$. Лемма доказана.

Пусть число $k_{3}-k_{3}^{(0)}$ мало и является чисто мнимым. Тогда в силу лемм 2,3 оператор $A(k)$ самосопряжен (из справедливости равенства $(A(k) \phi, \psi)-(\phi, A(k) \psi)=0$ в лакуне в силу теоремы единственности вытекает его справедливость во всей окрестности точки $\left.k_{3}^{(0)}\right)$. Обозначим через $\lambda_{n}(k) \in \mathbb{R}$ и $\phi_{n}(x, k), n=1,2, \ldots$, собственные значения $A(k)$, занумерованные в порядке невозрастания модуля с учетом кратности, и соответствующие им собственные функции, образующие ортонормированньй базис в $L^{2}(\Omega)$. Согласно теории возмущений (см. [4], добавление 1$)$ как $\lambda_{n}(k)$, так и $\phi_{n}(x, k)\left(\right.$ как $L^{2}(\Omega)$ значные функции) можно считать аналитическими по переменной $\kappa_{3}=-i\left(k_{3}-k_{3}^{(0)}\right)$ в некоторой вешественной окрестности нуля.

Введем обозначение $\phi_{0}(x)=\sqrt{W(x)} \psi_{n_{0}}\left(x, k_{0}\right)$. Далее предполагаем, что существует ограниченный обратный оператор $\left(1+A\left(k_{0}\right)\right)^{-1}$; в силу теоремы Фредгольма это эквивалентно тому, что $\lambda_{n}\left(k_{0}\right) \neq-1$ для всех $n=1,2, \ldots$ (см. ниже, в начале раздела 3 обсуждение, касающееся выполнения данного условия).

Положим $c_{n}(k)=\left(\phi_{0}(x), \phi_{n}(x, k)\right), n=1,2, \ldots$.

Лемма 4. Пусть $H$ - гильбертово пространство, $A=A_{1}+a\left(\cdot, x_{0}\right) y_{0}$, әде $A_{1}-$ линейный ограниченный оператор, действующий в $H$, такой, что существует обратный оператор $\left(1+A_{1}\right)^{-1}, \quad a=\mathrm{const} \neq 0, x_{0}, y_{0} \in H \backslash\{0\}$. Тогда обратный оператор $(1+A)^{-1}$ существует в том и только в том случае, если

$$
1+a\left(\left(1+A_{1}\right)^{-1} y_{0}, x_{0}\right) \neq 0
$$

ДокАЗАТЕЛЬСТво. Имеем

$$
1+A=\left(1+A_{1}\right)\left(1+a\left(\cdot, x_{0}\right)\left(1+A_{1}\right)^{-1} y_{0}\right)
$$

Достаточно найти условие обратимости второго сомножителя в правой части (9). Положим для $x \in H$

$$
y=x+a\left(x, x_{0}\right)\left(1+A_{1}\right)^{-1} y_{0} .
$$

Имеем $x=C\left(1+A_{1}\right)^{-1} y_{0}+y$, где $C=$ const. Подставляя данное выражение в (10), получаем равенство

$$
C\left(1+A_{1}\right)^{-1} y_{0}+C a\left(\left(1+A_{1}\right)^{-1} y_{0}, x_{0}\right)\left(1+A_{1}\right)^{-1} y_{0}=-a\left(y, x_{0}\right)\left(1+A_{1}\right)^{-1} y_{0} .
$$

Отсюда находим, что условие однозначной разрешимости уравнения (10) относительно $x$ для любого $y$ или, что то же, условие сушествования оператора $(1+A)^{-1}$, совпадает с (8), что и доказывает лемму. 
Tеорема 1. Пусть поверхность уровней проходит через точку $k_{0}$. Тогда справедливо равенство

$$
\sum_{n=1}^{\infty} \frac{\left|c_{n}\left(k_{0}\right)\right|^{2}}{1+\lambda_{n}\left(k_{0}\right)}=0
$$

ДоказательСтво. В силу (3) имеем $\left(k \neq k_{0}\right)$

$$
\sqrt{W} R_{0}(k) \sqrt{W}=-\frac{\operatorname{sign}(W)\left(\cdot, \phi_{0}\right) \phi_{0}}{i\left(k_{3}-k_{3}^{(0)}\right) \partial^{2} E_{n_{0}}\left(k_{0}\right) / \partial k_{3}^{2}}+A(k) .
$$

Согласно лемме 4 получаем условие несуществования оператора $\left(1+\sqrt{W} R_{0}(k) \sqrt{W}\right)^{-1}$ или, что то же, условие сушествования уровня в окрестности точки $k_{0}$ :

$$
\Delta(k)=i\left(k_{3}-k_{3}^{(0)}\right) \frac{\partial^{2} E_{n_{0}}\left(k_{0}\right)}{\partial k_{3}^{2}}-\operatorname{sign}(W)\left((1+A(k))^{-1} \phi_{0}, \phi_{0}\right)=0 ;
$$

$\mathrm{HO}$

$$
\left((1+A(k))^{-1} \phi_{0}, \phi_{0}\right)=\left(\sum_{n=1}^{\infty} \frac{\left(\phi_{0}(x), \phi_{n}(x, k)\right) \phi_{n}(x, k)}{1+\lambda_{n}(k)}, \phi_{0}(x)\right)=\sum_{n=1}^{\infty} \frac{\left|c_{n}(k)\right|^{2}}{1+\lambda_{n}(k)} .
$$

По условию существует последовательность точек $k_{n} \in \mathcal{V}, n=1,2, \ldots$, таких, что $k_{n} \rightarrow k_{0}, n \rightarrow \infty$. Из (12) и (13) для $k=k_{n}$, переходя к пределу при $n \rightarrow \infty$, получаем (11). Теорема доказана.

\section{3. СЛУЧАЙ ВОЗМУШЕНИЯ, ЗАВИСЯЩЕГО ОТ ПАРАМЕТРА}

В дальнейшем вместо $W(x)$ рассматриваем зависящую от параметра функцию $\mu W(x), \mu \in \mathbb{R}$. Это приводит к замене $\lambda_{n}$ на $\mu \lambda_{n}$ и $\phi_{0}$ на $\sqrt{\mu} \phi_{0} ;$ при этом $\phi_{n}, n=1,2, \ldots$, остаются теми же. При этом легко добиться выполнения условия $\mu \lambda_{n}\left(k_{0}\right) \neq-1, n=$ $1,2, \ldots$, сколь угодно малым изменением $\mu$, в дальнейшем предполагается выполнение этого условия.

Положим (см. (12))

$$
\Delta(k, \mu)=i\left(k_{3}-k_{3}^{(0)}\right) \frac{\partial^{2} E_{n_{0}}\left(k_{0}\right)}{\partial k_{3}^{2}}-\operatorname{sign}(\mu W)\left((1+\mu A(k))^{-1} \phi_{0}, \phi_{0}\right) .
$$

В следуюшей теореме 2 будем предполагать, что для всех $\mu \quad \Delta(k, \mu) \not \equiv 0$ на многообразии $\mathcal{V}$ или, что то же самое, дробь $\Delta(k, \mu) /\left(k_{3}-k_{3}^{(0)}\right)$ несократима. (Заметим по поводу этого предположения, что возмушение $\mu W(x)$, главным образом определяющее уровни $\mathcal{V}$, т.е. нули функции $\Delta(k, \mu)$, и потенциал $V(x)$, определяюший множество $\mathcal{V}_{0}$, не связаны друг с другом. См. также замечание 2 к теореме 2 , в котором сформулирован вариант этой теоремы, не требующий данного предположения.)

Введем обозначение

$$
a_{n}=\sum_{m: \lambda_{m}\left(k_{0}\right)=\lambda_{n}\left(k_{0}\right)}\left|c_{m}\left(k_{0}\right)\right|^{2} .
$$


TЕорема 2. Пусть $0<\lambda_{n_{0}}\left(k_{0}\right)<\lambda_{n_{0}^{\prime}}\left(k_{0}\right) \quad\left(\lambda_{n_{0}}\left(k_{0}\right)<\lambda_{n_{0}^{\prime}}\left(k_{0}\right)<0\right)-$ два соседних собственных значения оператора $A\left(k_{0}\right)$, причем $a_{n_{0}} \neq 0, a_{n_{0}^{\prime}} \neq 0$. Тогда существует единственное число $\mu_{0} \in\left(-1 / \lambda_{n_{0}}\left(k_{0}\right),-1 / \lambda_{n_{0}^{\prime}}\left(k_{0}\right)\right)$ такое, что у оператора $H_{\mu}\left(k_{\|}\right)=H_{0}\left(k_{\|}\right)+\mu W(x)$ поверхность уровней проходит через точку $k_{0}$.

ДоказАтЕльство. Рассмотрим для определенности случай $\lambda_{n_{0}}\left(k_{0}\right)<\lambda_{n_{0}^{\prime}}\left(k_{0}\right)<0$, тогда $\mu>0$. Докажем, что функция $\Delta\left(k_{0}, \mu\right)$ обрашается в нуль в некоторой точке $\mu_{0}$ интервала $\delta=\left(-1 / \lambda_{n_{0}}\left(k_{0}\right),-1 / \lambda_{n_{0}^{\prime}}\left(k_{0}\right)\right)$. Положим $\epsilon=-1 / \mu$, тогда в силу равенства (см. (13))

$$
\Delta\left(k_{0}, \mu\right)=-\mu \operatorname{sign}(W) \sum_{n=1}^{\infty} \frac{\left|c_{n}\left(k_{0}\right)\right|^{2}}{1+\mu \lambda_{n}\left(k_{0}\right)}
$$

и стремления к нулю последовательности собственных значений $\lambda_{n}\left(k_{0}\right)$ функция

$$
\begin{aligned}
& \Delta\left(k_{0}, \mu\right)+\mu \operatorname{sign}(W)\left(\frac{a_{n_{0}}}{1+\mu \lambda_{n_{0}}\left(k_{0}\right)}+\frac{a_{n_{0}^{\prime}}}{1+\mu \lambda_{n_{0}^{\prime}}\left(k_{0}\right)}\right)= \\
&=\Delta\left(k_{0},-\frac{1}{\epsilon}\right)+\operatorname{sign}(W)\left(\frac{a_{n_{0}}}{\lambda_{n_{0}}\left(k_{0}\right)-\epsilon}+\frac{a_{n_{0}^{\prime}}}{\lambda_{n_{0}^{\prime}}\left(k_{0}\right)-\epsilon}\right)
\end{aligned}
$$

равномерно ограничена по $\epsilon \in\left(\lambda_{n_{0}}\left(k_{0}\right), \lambda_{n_{0}^{\prime}}\left(k_{0}\right)\right)$. При возрастании $\epsilon$ от $\lambda_{n_{0}}\left(k_{0}\right)+0$ до $\lambda_{n_{0}^{\prime}}\left(k_{0}\right)-0$ выражение

$$
\frac{a_{n_{0}}}{\lambda_{n_{0}}\left(k_{0}\right)-\epsilon}+\frac{a_{n_{0}^{\prime}}}{\lambda_{n_{0}^{\prime}}\left(k_{0}\right)-\epsilon}
$$

а вместе с ним и функция $\Delta\left(k_{0},-1 / \epsilon\right)\left(\right.$ или $\left.-\Delta\left(k_{0},-1 / \epsilon\right)\right)$ возрастают от $-\infty$ до $\infty$. Отсюда вытекает, что в некоторой точке $\mu_{0} \in \delta$ имеет место равенство $\Delta\left(k_{0}, \mu_{0}\right)=0$. Равенство $\Delta\left(k, \mu_{0}\right)=0$ определяет уровни (см. доказательство теоремы 1$)$, следовательно, $\Delta\left(k, \mu_{0}\right) \not \equiv 0$ по переменной $k_{3}$, иначе $E_{0}$ не было бы границей зоны. Пусть $k_{3}^{(m)}\left(\tilde{k}_{\|}\right), m=1, \ldots, M,-$ корни многочлена Вейерштрасса по переменной $k_{3}$, отвечающего функции $\Delta\left(k, \mu_{0}\right)$ в окрестности точки $k_{0}[9]$. Очевидно, что множество нулей функции $\Delta\left(k, \mu_{0}\right)$ задается в окрестности $k_{0}$ равенствами $k_{3}=k_{3}^{(m)}\left(\tilde{k}_{\|}\right), m=1, \ldots, M$. Пусть равенство $k_{3}=k_{3}^{(0)}\left(\tilde{k}_{\|}\right)$определяет многообразие $\mathcal{V}_{0}$ (см. введение). В силу предположения, сформулированного перед теоремой 2 , в произвольно малой окрестности точки $k_{\|}$имеем $k_{3}^{(m)}\left(\tilde{k}_{\|}\right) \not \equiv k_{3}^{(0)}\left(\tilde{k}_{\|}\right), m=1, \ldots, M$, и потому $k_{0}$ принадлежит замыканию множества $\mathcal{V}$.

Докажем единственность $\mu$. Для этого вычислим

$$
\begin{aligned}
\frac{\partial \Delta\left(k_{0}, \mu\right)}{\partial \mu}= & -\operatorname{sign}(W)\left(\left((1+\mu A(k))^{-1} \phi_{0}, \phi_{0}\right)-\right. \\
& \left.-\mu\left((1+\mu A(k))^{-1} A(k)(1+A(k))^{-1} \phi_{0}, \phi_{0}\right)\right)= \\
= & -\operatorname{sign}(W)\left((1+A(k))^{-2} \phi_{0}, \phi_{0}\right) .
\end{aligned}
$$

Данная величина сохраняет знак, следовательно, функция $\Delta\left(k_{0}, \mu\right)$ строго монотонна по переменной $\mu$, и $\Delta\left(k_{0}, \mu\right) \neq 0$, если $\mu \neq \mu_{0}$, так что в силу теоремы 1 чисел $\mu \neq \mu_{0}$, для которых $\mathcal{V}$ проходило бы через точку $k_{0}$, не существует. Теорема доказана. 
ЗАмечАниЕ 1 . В силу компактности оператора $A\left(k_{0}\right)$ имеем $\lim _{n \rightarrow \infty} \lambda_{n}=0$. Если при этом число, например, положительных собственных значений бесконечно велико и для них всегда выполняются условия теоремы, то при $\mu \rightarrow-\infty$ поверхности уровней систематически бесконечное число раз проходят через границу зоны.

ЗАмечАниЕ 2. Аналогично с использованием равенства $\Delta(k, \mu)=0$ без наложения условия на множества $\mathcal{V}$ и $\mathcal{V}_{0}$ можно доказать, что любая точка $k$ вблизи $k_{0}$ такая, что $k_{3}-k_{3}^{(0)} \in i \mathbb{R}$, является уровнем для некоторого $\mu_{0} \in\left(-1 / \lambda_{n_{0}}\left(k_{0}\right),-1 / \lambda_{n_{0}^{\prime}}\left(k_{0}\right)\right)$, определяемого однозначно.

В силу сказанного выше множество $\mathcal{V}$ по переменной $k$ описывается нулями функции $\Delta(k, \mu)$, которые представляют собой корни многочлена Вейерштрасса по переменной $k_{3}$, имеюшие в силу теоремы Пюизо [1] вид

$$
k_{3}\left(k_{\|}, \mu\right)=k_{3}^{(0)}\left(k_{\|}, \mu\right)+\sum_{n=1}^{\infty} \alpha_{n}\left(k_{\|}\right)\left(\mu-\mu_{0}\right)^{n / p}
$$

где $p \geqslant 1$ - некоторое целое число (выбрана одна из ветвей корня $\left(\mu-\mu_{0}\right)^{1 / p}$ ).

Будем говорить, что уровни, отвечаюшие корню $k_{3}\left(k_{\|}, \mu\right)$ многочлена Вейерштрасса, заходят в лакуну, если для всех $\mu>\mu_{0}$ (или $\mu<\mu_{0}$ ), достаточно близких к $\mu_{0}$, значения $E$, соответствуюшие точкам $k_{3}=k_{3}\left(k_{\|}, \mu\right)$ из окрестности $k_{3}^{(0)}$, принадлежат лакуне и являются собственными значениями. (Число $E$, будучи на втором листе, может находиться в лакуне, но не быть собственным значением.)

Пусть $\mu_{0} \neq 0, \mu_{0} \lambda_{n}\left(k_{0}\right) \neq-1$. Заметим, что функция

$$
f(k, \mu)=\operatorname{sign}(\mu W)\left((1+\mu A(k))^{-1} \phi_{0}, \phi_{0}\right)=\operatorname{sign}(W) \sum_{n=1}^{\infty} \frac{\mu\left|c_{n}(k)\right|^{2}}{1+\mu \lambda_{n}(k)}
$$

в силу теоремы Хартогса [9] аналитична по совокупности переменных в окрестности точки $\left(\mu_{0}, k_{0}\right)$.

ТЕОРема 3. Предположим, что уровни, отвечающие корню $k_{3}=k_{3}\left(k_{\|}, \mu\right)$, заходят в лакуну. Функция $k_{3}\left(k_{\|}, \mu\right)$ является аналитической функиией параметра $\mu$ в окрестности $\mu_{0}$ в том и только в том случае, если

$$
-1+\frac{\frac{\partial f\left(k_{0}, \mu_{0}\right)}{\partial k_{3}}}{i \frac{\partial^{2} E_{n_{0}}}{\partial k_{3}^{2}}} \neq 0
$$

При этом

$$
\frac{\partial \operatorname{Im} k_{3}\left(k_{\|}, \mu_{0}\right)}{\partial \mu} \neq 0
$$

и при переходе параметра через точку $\mu_{0}$ собственное значение становится резонансом. 
ДокАЗАТЕльство. Пусть $k_{3}=k_{3}^{(0)}+i \kappa_{3}$, где $\kappa_{3} \in \mathbb{R}$. С использованием выражения для функции $f(k, \mu)$ перепишем равенство (12) для $k \in \mathcal{V}$ с $\mu W$ вместо $W$ :

$$
-i\left(k_{3}-k_{3}^{(0)}\right) \frac{\partial^{2} E_{n_{0}}\left(k_{0}\right)}{\partial k_{3}^{2}}+f(k, \mu)=0 .
$$

Предположим, что выполнено (15), тогда по теореме о неявной функции [9] уравнение $(16)$ определяет в комплексной окрестности точки $\left(k_{3}^{(0)}, \mu_{0}\right)$ комплексно-аналитическое многообразие, описываемое аналитической функцией $k_{3}=\tilde{k}_{3}(\mu)$. С другой стороны, функция $k_{3}=k_{3}\left(k_{\|}, \mu\right)=k_{3}(\mu)$, описываюшая уровни, заходяшие в лакуну, для $\mu>\mu_{0}$ (или для $\left.\mu<\mu_{0}\right)$ в силу лемм 2,3 удовлетворяет уравнению (16). Полагая для определенности $\mu>\mu_{0}$, согласно (14) имеем

$$
\begin{aligned}
k_{3}(\mu)=\tilde{k}_{3}(\mu) & =k_{3}^{(0)}\left(k_{\|}, \mu\right)+\sum_{n=1}^{\infty} \alpha_{n}\left(k_{\|}\right)\left(\mu-\mu_{0}\right)^{n / p}= \\
& =k_{3}^{(0)}\left(k_{\|}, \mu\right)+\sum_{m=1}^{\infty} \tilde{\alpha}_{m}\left(k_{\|}\right)\left(\mu-\mu_{0}\right)^{m},
\end{aligned}
$$

где в правой части стоит ряд Тейлора функции $\tilde{k}_{3}(\mu)$. Из равенства рядов легко получаем, что в разложении по степеням $\left(\mu-\mu_{0}\right)^{1 / p}$ отличны от нуля лиш коэффициенты с номерами, кратными $p$, и, следовательно, функция $k_{3}(\mu)$ является аналитической.

С другой стороны, пусть уровни, отвечающие корню $k_{3}(\mu)$, заходят в лакуну, и функция $k_{3}(\mu)$ является аналитической. Подставив в $(16) k_{3}=k_{3}(\mu)$, дифференцируя по $\mu$ и переходя к пределу при $\mu \rightarrow \mu_{0}$, получаем

$$
\left(-i \frac{\partial^{2} E_{n_{0}}\left(k_{0}\right)}{\partial k_{3}^{2}}+\frac{\partial f\left(k_{0}, \mu_{0}\right)}{\partial k_{3}}\right) k_{3}^{\prime}\left(\mu_{0}\right)+\frac{\partial f\left(k_{0}, \mu_{0}\right)}{\partial \mu}=0 ;
$$

HO

$$
\frac{\partial f\left(k_{0}, \mu_{0}\right)}{\partial \mu}=\operatorname{sign}(W) \sum_{n=1}^{\infty} \frac{\left|c_{n}\left(k_{0}\right)\right|^{2}}{\left(1+\mu_{0} \lambda_{n}\left(k_{0}\right)\right)^{2}} \neq 0
$$

(см. доказательство теоремы 2). Из (17), (18) и вешественности функции $f$ вытекают неравенство $(15)$ и неравенство $k_{3}^{\prime}\left(\mu_{0}\right) \neq 0$, а также включение $k_{3}^{\prime}\left(\mu_{0}\right) \in i \mathbb{R}$. Из этого включения и равенства

$$
k_{3}(\mu)=k_{3}^{(0)}+k_{3}^{\prime}\left(\mu_{0}\right)\left(\mu-\mu_{0}\right)+o\left(\mu-\mu_{0}\right)
$$

следует, что $\operatorname{Im} k_{3}(\mu)$ меняет знак при переходе через $\mu_{0}$, что и доказывает последнее утверждение теоремы.

ЗАмЕчАнИЕ 1 . Пусть для определенности $E_{0}$ - нижняя гранища зоны. При переходе параметра $\mu$ через точку $\mu_{0}$ собственное значение, возрастая до границы зоны $E_{0}$, в точке $\mu_{0}$ "отражается" от $E_{0}$ и далее, оставаясь вешественным, убывает, переходя на второй лист и преврашаясь в резонанс (в “виртуальный уровень" (см. [10], гл. 13)). 
ЗАмечАнИЕ 2 . Из условия $k_{3}^{\prime}\left(\mu_{0}\right) \neq 0$ вытекает, что в $(14) \alpha_{1}\left(k_{\|}\right) \neq 0$ при $p=1$. Если теперь для некоторого $p>1$ выполнено $\alpha_{1}\left(k_{\|}\right) \neq 0$ и уровни, отвечаюшие $k_{3}(\mu)$, заходят в лакуну, то при переходе $\mu$ через $\mu_{0}$ собственное значение и в этой ситуации становится резонансом. Действительно, в противном случае выражение $\phi(\mu)=$ $i \alpha_{1}\left(k_{\|}\right)\left(\mu-\mu_{0}\right)^{1 / p}$, будучи вешественным, сохраняло бы знак в окрестности точки $\mu_{0}$, но тогда разность $\mu-\mu_{0}=\left(\phi(\mu) / i \alpha_{1}\left(k_{\|}\right)\right)^{p}$ тоже должна сохранять знак в окрестности $\mu_{0}$, получаем противоречие. Переход собственного значения в собственное значение может быть, например, в ситуации, когда $k_{3}(\mu)=k_{3}^{(0)}+i c_{2}\left(\mu-\mu_{0}\right)^{2 / p}+o\left(\left(\mu-\mu_{0}\right)^{2 / p}\right)$, где $c_{2}>0$, и корень вешественный.

\section{Список литературы}

[1] М. Рид, Б. Саймон. Методы современной математической физики. Т. 4. Анализ операторов. М.: Мир, 1982

[2] E. B. Davies. Proc. Cambridge Philos. Soc. 1977. V. 82. P. 327-334.

[3] Ю. П. Чубурин. ТМФ. 1997. Т. 110. № 3. С. 443-453.

[4] Ф. А. Березин, М. А. Шубин. Уравнение Шредингера. М.: Изд-во МГУ, 1983.

[5] С. Алъбеверио, Ф. Гестези, Р. Хёэг-Крон, Х. Холъден. Решаемые модели в квантовой механике. М.: Мир, 1991.

[6] А. А. Арсенъев. ТМФ. 1995. Т. 104. № 2. С. 214-232.

[7] Г. В. Волъф, Ю. П. Чубурин, Л. А. Рубцова. Поверхность. 1991. № 10. С. 81-89.

[8] М. ШШ. Бирман. Алгебра и анализ. 1996. Т. 8. № 1. С. 3-20.

[9] Р. Ганнинг, Х. Росси. Аналитические функции многих комплексных переменных. М.: Мир, 1969.

[10] Дж. Тейлор. Теория рассеяния. Квантовая теория нерелятивистских столкновений. М.: Мир, 1975.

Поступила в редакцию 17.I.2000 г., после доработки 18.VIII.2000 г. 